Bond University

Research Repository

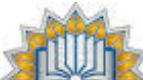 \\ Bovo \\ UNIVERSITY
}

\section{Effect of high polyphenol extra virgin olive oil on markers of cardiovascular disease risk in healthy Australian adults (OLIVAUS): A protocol for a double-blind randomised, controlled, cross-over study}

Marx, Wolfgang; George, Elena S.; Mayr, Hannah L.; Thomas, Colleen J.; Sarapis, Katerina; Moschonis, George; Kennedy, Greg; Pipingas, Andrew; Willcox, Jane C.; Prendergast, Luke A.; Itsiopoulos, Catherine

Published in:

Nutrition and Dietetics

DOI:

$10.1111 / 1747-0080.12531$

Licence:

Other

Link to output in Bond University research repository.

Recommended citation(APA):

Marx, W., George, E. S., Mayr, H. L., Thomas, C. J., Sarapis, K., Moschonis, G., Kennedy, G., Pipingas, A., Willcox, J. C., Prendergast, L. A., \& Itsiopoulos, C. (2020). Effect of high polyphenol extra virgin olive oil on markers of cardiovascular disease risk in healthy Australian adults (OLIVAUS): A protocol for a double-blind randomised, controlled, cross-over study. Nutrition and Dietetics, 77(5), 523-528. https://doi.org/10.1111/17470080.12531

\section{General rights}

Copyright and moral rights for the publications made accessible in the public portal are retained by the authors and/or other copyright owners and it is a condition of accessing publications that users recognise and abide by the legal requirements associated with these rights.

For more information, or if you believe that this document breaches copyright, please contact the Bond University research repository coordinator. 


\section{$1 \quad$ Abstract}

2 Background: Previous clinical studies have suggested that high polyphenol extra virgin

3 olive oil provides a superior cardioprotective effect compared to low polyphenol olive

4 oil. However, further studies are required to replicate these results in non-Mediterranean

5 populations.

6 Aim: To investigate the effect of high polyphenol extra virgin olive oil vs low polyphenol

7 olive oil with known polyphenol composition on markers of cardiovascular disease risk

8 in a healthy non-Mediterranean cohort.

9 Method: In a double-blind randomised cross-over trial, this study will examine the effect

10 of high polyphenol extra virgin olive oil versus low-polyphenol olive oil in 50 healthy

11 participants. Each intervention phase will be 3 weeks long with a 2-week washout period

12 between each phase. Outcomes to be assessed include HDL cholesterol efflux, oxidized-

13 LDL, blood lipids, C-reactive protein, arterial stiffness, blood pressure, and cognitive

14 function. Dietary intake, physical activity levels and anthropometry will also be collected.

15 Discussion: Due to the rigorous trial design, novel and clinically relevant outcomes, the

16 use of a well-characterised extra virgin olive oil, and, in contrast to the current literature,

17 the non-Mediterranean study population, this study will provide a significant contribution

18 to the understanding of the clinical importance of polyphenol intake in the Australian

19 socio-cultural context.

20 Keywords: olive oil, cognition, oxidative stress, polyphenol, biophenol, Mediterranean 21 diet, 


\section{$1 \quad 1 \quad$ Introduction}

2 The traditional Mediterranean diet, known for its cardioprotective effect, has been shown

3 to improve cardiovascular disease risk factors including specific measures of blood lipids

4 (HDL cholesterol, triglycerides), markers of inflammation, blood pressure, fasting blood

5 glucose and risk of diabetes. ${ }^{1,2}$ The traditional Mediterranean diet is characterised by an

6 abundance of plant foods (e.g. leafy greens, tomatoes, onions, herbs, wholegrain cereals,

7 legumes and nuts), moderate amounts of fermented dairy foods, seafood, red wine and

8 small quantities of red meat and home-made sweets..$^{3-5}$ Of particular relevance to the

9 proposed study, is the large servings of extra virgin olive oil (EVOO; 60-80ml daily) as

10 the primary source of culinary fat and a unique culinary component of the Mediterranean

11 dietary pattern. Olive oil contains highly variable concentrations of polyphenols which

12 can be affected by season, olive variety, region and soil, ripeness of the fruit and

13 processing. ${ }^{6}$ EVOO is characterised by a low-temperature, mechanical processing

14 technique which preserves the higher polyphenol content in comparison to the refining

15 methods such as deodorization and chemical processing techniques used to produce

16 refined olive oils, which subsequently have significantly lower polyphenol content. ${ }^{7,8}$

17 In healthy adults, EVOO has been shown to improve CVD risk factors including blood

18 pressure, low grade inflammation, and lipid profile. ${ }^{9}$ The cardioprotective properties of

19 EVOO have been primarily attributed to the high monounsaturated fat content; however,

20 EVOO contains an array of unique polyphenols, also referred to as "biophenols". ${ }^{10}$ These

21 polyphenols have shown improvements in measures of glucose metabolism, lipid

22 peroxidation, and cholesterol markers in clinical trials. ${ }^{11-14}$ Despite this evidence, the

23 unique, cardioprotective polyphenols in EVOO are not currently recognised by 
1 cardiovascular disease guidelines, possibly due to the need for additional high-level

2 evidence.

3 To further understand the mechanisms involved in the cardioprotective effect of EVOO-

4 derived polyphenols, further clinical research is needed to: (1) replicate previously

5 reported improvements in routinely measured cardiovascular markers (e.g. HDL/LDL

6 cholesterol, blood pressure) in the Australian population, (2) determine the feasibility of

7 a provision of $60 \mathrm{ml}$ of EVOO per day in a non-Mediterranean population, and (3)

8 investigate the effect of high-polyphenol EVOO on novel CVD risk markers. Increased

9 CVD risk has, in part, been attributed to low plasma levels of HDL cholesterol (HDL-

10 C). ${ }^{15}$ However, emerging evidence suggests that impaired HDL function, rather than low

11 HDL-C, may explain HDL-associated CVD risk. ${ }^{16}$ HDL-C efflux, as measure of HDL

12 function, has been identified as a marker that may independently predict risk of CVD. ${ }^{17}$

13 To improve the existing evidence-base in this area, the proposed trial aims to investigate

14 the effect of a high-polyphenol EVOO compared to a low-polyphenol olive oil on both

15 routinely measured (e.g. blood pressure and cholesterol) and novel markers (e.g. HDL-

16 cholesterol efflux) on CVD risk in a healthy Australian cohort.

17 Furthermore, recently published clinical and animal studies have provided preliminary

18 evidence to suggest that EVOO, as well as other polyphenol-rich interventions, may

19 improve cognitive performance and prevent age- or experimentally-induced cognitive

20 impairment. ${ }^{18,19}$ Hence, as a secondary outcome, this study will also investigate the effect

21 of high polyphenol EVOO and low polyphenol olive oil on measures of cognitive

22 performance in this healthy cohort. 
1 The OLIVAUS study is a double-blind, randomised, controlled cross-over trial that aims

2 to investigate the effect of a 3-week intervention of high polyphenol EVOO compared to

3 a retail-purchased low polyphenol olive oil on CVD risk factors in 50 healthy participants

4 (Fig. 1). Compared with a low polyphenol olive oil, we hypothesise that a high polyphenol

5 EVOO intervention will result in improved measures of HDL cholesterol efflux, oxidised

6 LDL and low-grade inflammation in a healthy adult population. The trial protocol

7 (registered 30/04/2018, updated 13/02/2019) has been prospectively registered with the

8 Australia New Zealand Clinical Trials Registry <removed> and was created in

9 accordance with the SPIRIT Statement. ${ }^{20}$

10 This trial will be conducted in accordance with the Guidelines for Good Clinical Practice

11 and the Declaration of Helsinki and CONSORT reporting guidelines. The trial team has

12 obtained written approval for the protocol and Patient Information and Consent Form

13 from the <removed> University Human Research Ethics Committee (HEC17-067).

14 Participants will be recruited in Melbourne, Australia using social media advertisements,

15 and through <removed> University using email advertisements, mailing lists, word of mouth, and posters on campus and at local medical clinics. Table 1 provides the inclusion

17 and exclusion criteria for this study.

18 Figure 1 provides a visual representation of the study flow. The participant schedule 19 throughout the trial is shown in Table 2, including data collection time-points. Once enrolled, participants will be asked to undergo an initial washout period where they will

21 be instructed to abstain from consuming all olive oil, olive products, and antioxidant 22 supplements for two weeks prior to the scheduled baseline meeting (T1). Participants will 23 be requested to complete a 3-day diet diary including 2 week days and one weekend day 24 where they are asked to include details on the foods and beverages consumed including 
1 type, brand, quantity in household measures and cooking methods. Participants will be

2 asked to complete this diet diary in the days preceding the initial appointment and at the

3 conclusion of the intervention phases. Participants will be asked to come to the baseline

4 meeting in a fasted state. At the end of each intervention phase, participants will receive

5 a \$25AUD gift voucher (\$50AUD in total).

6 The research staff will screen against the eligibility criteria during a face-to-face meeting.

7 Following informed consent, participant numbers will be assigned sequentially and will

8 be block randomised to receive either high polyphenol EVOO or low polyphenol olive

9 oil. The block randomisation sequence will be developed using blocks of 6 , by a senior

10 researcher $(<$ removed $>$ ), who will not have any direct involvement in the participant

11 recruitment or data collection phase. After baseline measures are taken, a researcher who

12 is not involved in any participant contact (<removed >) will email the allocation for each

13 participant to the team. De-identified bottles of high and low polyphenol olive oil will be

14 randomised and coded prior to the recruitment phase and all staff will be blinded to this

15 randomisation.

16 Participants will receive a 3-week supply of the either the low polyphenol olive oil or 17 high-polyphenol EVOO (1.26 litres) at the commencement of the first intervention (T1) 18 and the commencement of the second intervention (T4). Participants will be required to 19 consume $60 \mathrm{ml}$ per day for each of the 3-week intervention phases. Measuring cups will 20 be provided for participant use, where appropriate, to demonstrate the required volume.

21 Emphasis on strategies that incorporate olive oil into their habitual diet in a raw, uncooked

22 form will be provided by researchers. This will include dressing salads or vegetables,

23 drizzling the oil on prepared meals such as soups or casseroles, and ensuring leftover 
1 amounts are also consumed. Participants will be supplied with the full amount of EVOO

2 and olive oil required per 3-week intervention period.

3 Total polyphenol and polyphenol subclasses for each olive oil intervention were analysed

4 by Modern Olives Laboratory Services (Lara, VIC), a Commonwealth Government

5 accredited testing agency, using high-performance liquid chromatography (HPLC).

6 Samples were prepared and blinded for the researcher. Table 3 provides a comparison of

7 the total polyphenol and polyphenol subclasses of each olive oil intervention. All high

8 polyphenol EVOO was sourced from Cobram Estate Pty. Ltd. from the same harvest and

9 lot and stored under the same conditions. An EVOO with a confirmed polyphenol count

10 of approximately $320 \mathrm{ppm}$ will be provided to participants as the high polyphenol EVOO

11 intervention. A low polyphenol olive oil was sourced from a local supermarket where a

12 bulk purchase of the same brand from the same lot number was made. This oil was

13 confirmed to have a polyphenol count of approximately $86 \mathrm{ppm}$.

14 At the commencement of the first and second intervention phase meeting (T1 and T4),

15 participants will attend a one-hour appointment in the morning with the research staff at

16 the nutrition clinical rooms, <removed>campus, <removed> University. Data collection

17 including three-day diet diaries, medical history and lifestyle (e.g. physical activity)

18 questionnaires, anthropometry, fasting blood collection, blood pressure, arterial stiffness

19 measures and cognitive performance will take place at each face to face appointment.

20 Basic demographic data will also be collected at baseline including age, gender, and

21 ethnicity. These are described in further detail below. 
1 The research staff will contact participants by phone or email approximately 1.5 weeks

2 into each intervention phase to discuss progress, adherence to the intervention and to ask

3 participants if they have experienced any adverse events during the study period.

4 At the end of each intervention phase (T3 and T6) participants will attend a face to face

5 appointment where they will complete all the data collection indicated at the T1 and T3

6 appointment. In addition, participants will be required to return their olive oil bottles so

7 that research staff can record the weight of any remaining oil as an additional marker of

8 adherence.

9 For T3 only: Research staff will instruct the participants to undergo a 2-week washout 10 period whereby they cease consumption of all olive oil and olive products during this 11 period, until their next meeting (T4, start of second olive oil phase).

12 For T6 only: Research staff will assess blinding by asking the participant about the order

13 they think they received the two olive oil interventions and whether there were any 14 differences in taste.

15 All outcomes described below will be measured pre and post the olive oil intervention 16 phases (T1, T3, T4, T6) as per Table 2. Blood collection will also take place at each pre 17 and post time point. Research staff will confirm that participants have fasted for 8-12 18 hours. If so, fasting venous blood samples will be obtained, by a researcher trained in 19 venepuncture, from the antecubital vein using standard venous puncture techniques. If 20 blood collection is unsuccessful research staff will arrange for blood collection at a local 21 pathology centre within 48 hours of the scheduled appointment.

22 HDL cholesterol efflux, the primary outcome, will be analysed using a Cholesterol Efflux

23 Fluorometric Assay Kit (Biovision, USA). Participants will be invited to participate in an 
1 optional cognitive performance assessment. If they have consented to this aspect of the

2 trial, the participant will conduct the full cognitive assessment at each face to face

3 appointment. The Swinburne University Computerised Cognitive Assessment Battery

4 (SUCCAB) is a validated, computer based cognitive battery, administered using a 5-

5 button control box. ${ }^{21}$ Eight tests of cognitive function will be assessed by both accuracy

6 and response time. These tests include Simple and Choice Reaction Times, Immediate

7 and Delayed Recognition, Congruent and Incongruent Stroop colour-words, Spatial

8 Working Memory and Contextual Memory. This battery has been used in numerous

9 studies to assess the cognitive effects of dietary supplementation and other

10 interventions. $^{22-24}$

11 Total, HDL, and LDL cholesterol, high sensitivity C-reactive protein and triglyceride

12 levels will be measured using standard enzyme assays. Oxidized LDL will also be

13 analysed using a solid phase two-site enzyme immunoassay (ELISA; Mercodia ${ }^{\mathrm{TM}}$,

14 Sweden).

15 Cardiovascular function will be assessed using the non-invasive SphygomoCor XCEL

16 system (AtCor Medical, Australia) once the participant has rested for five minutes in the

17 supine position. Assessments will include standard brachial blood pressures, aortic

18 (central) blood pressures, pulse wave analysis of peripheral arterial stiffness, and carotid-

19 femoral pulse wave velocity analysis of central arterial stiffness.

20 Three-day diet diaries will be collected at each face to face appointment. Research staff

21 will conduct a baseline interview with all participants and will confirm that they

22 underwent the required 2-week washout period. The research staff will also review the 3-

23 day dietary intake data to ensure sufficient detail has been recorded for nutrient analysis 
1 and to clarify any missing data on responses that look inaccurate. Participants will self-

2 report details regarding their intake of food and liquids over a three-day period including

3 the quantity (via household measures), type, and timing of items consumed. Furthermore,

4 a specific section to capture timing and amount of olive oil will be incorporated.

5 Participant weight, height and waist circumference will be measured using standard

6 techniques, in duplicate by the research staff. If there is $>10 \%$ variation between the two

7 measures, a third measure will be obtained. The mean of the closest two measures will be

8 used. Self-reported physical activity will be completed prior to the commencement of the

9 trial (T1) and at the end of the trial (T6) via the Active Australia Survey, ${ }^{25}$ a validated

10 tool within the Australian population and consists of 8 questions to assess the previous 7

11 days. The questionnaire captures a range of activity types including walking, work in the

12 yard, vigorous physical activity and moderate physical activity. Adverse events will be

13 monitored at all time points. If a participant experiences significant adverse events, they

14 will be withdrawn from the study. All adverse events will be reported to the trial steering

15 committee, comprised of the Principal Investigator and trial staff. The Human Research

16 Ethics Committee will also be notified, as appropriate. Emergency unblinding will occur

17 for serious adverse events deemed related to the study product. All participant data will

18 be securely stored either in onsite locked cabinets or password protected documents on

19 secured university servers with restricted access to the study team only.

20 All outcomes will be analysed by using linear mixed-effects (LME) models with random 21 intercepts and slopes to account for within-participant correlation over time and varying 22 treatment effect amongst participants. The effect of intervention order, due to potential 23 carry-over effect, on all outcomes will be tested and adjusted for in the LME model if 24 necessary by including and interaction term between the treatment and period effects. A 
1 senior statistician (<removed >) will oversee the fitting of the LME models and be

2 responsible for assessing model validity.

3 Participant 3-day dietary records will be analysed and dietary changes will be used as a

4 covariate. Adjusted results will be calculated using a multiple linear regression model

5 including the stratification factors (e.g. gender, physical activity levels). A sensitivity

6 analysis comparing the LME analyses and pooled estimates from the multiple imputation

7 procedures will be conducted to prevent against bias. All reported $P$-values will be 2-

8 tailed. The levels of statistical significance will be set at $\mathrm{P}<0.05$ and estimates will be

9 accompanied with 95\% confidence intervals. All statistical analyses will be conducted

10 using the SPSS statistical software for Windows (version 25). Based on the results of

11 previous research, a sample size of 40 was considered adequate to provide sufficient

12 statistical power to detect a statistically significant 5\% difference in HDL cholesterol

13 efflux between the two intervention phases with $80 \%$ power and $5 \%$ level of

14 significance. ${ }^{26}$ To account for a $20 \%$ level of potential attrition, this sample size was

15 expanded to 50 participants.

\section{Results}

17 Recruitment commenced in July 2018 and is expected to be completed by late- 2019 .

18 Currently, a total of $\mathrm{n}=21$ participants have been enrolled in this trial, leading to an

19 average recruitment rate of 7 per month. Sixty five percent of participants are female with

20 a mean age of 37 years. Five of the currently recruited cohort have completed the

21 intervention with $100 \%$ of outcome data collected. Incomplete data have been collected

22 on one participant due to withdrawal from the study due to inability to consume the

23 required amount of olive oil. Ten percent of participants that have completed the 
1 intervention consumed at least $80 \%$ of the provided oils. There have been no reported

2 serious adverse events related to the study intervention. Reported adverse events include

3 diarrhoea, bloating, reflux and heartburn.

\section{Discussion}

5 Previous clinical studies have reported that EVOO provides a cardioprotective effect

6 through mediating improvements in cardiovascular risk factors, ${ }^{1,9}$ however, few studies

7 have investigated the contribution of the polyphenol component of olive oil to these

8 improvements. This study will compare the effect of high-polyphenol EVOO to low-

9 polyphenol olive oil on markers of CVD risk that are related to cholesterol transport and

10 metabolism, LDL oxidation, blood pressure (peripheral and central), arterial stiffness, and

11 inflammation, as well as measures of cognitive function. By implementing a study design

12 that will be able to differentiate between the effect of polyphenols from the other

13 components of olive oil (e.g. monounsaturated fat will remain consistent between study

14 arms), this trial will provide important information regarding the effect of EVOO

15 polyphenols on a range of cardiovascular risk factors and cognition. In contrast to the

16 current literature which has predominantly been conducted within Mediterranean

17 populations, this will assess the use of high-polyphenol EVOO in the Australian western

18 socio-cultural context. In addition, previous research has primarily assessed the effect of

19 a Mediterranean diet and EVOO in populations with existing co-morbidities such as

20 coronary heart disease, type 2 diabetes, cancer and cognitive decline while this study aims

21 to recruit healthy participants. ${ }^{2,27}$ This study is one of the first trials to comprehensively

22 assess the polyphenol composition within each of the oils provided to participants. Other

23 studies, even those which compare oils with varying polyphenol content, do not report 
1 the composition of the polyphenols contained within. ${ }^{9}$ Finally, this study will report HDL

2 efflux, oxidised LDL and other biomarkers of CVD that have not been extensively studied

3 in previous dietary intervention studies. If shown to be beneficial, this study will provide

4 evidence for a widely-accessible, low-cost dietary intervention to reduce cardiovascular

5 disease risk and will significantly contribute to the existing literature on the clinical

6 importance of polyphenol intake.

References

10 1. Estruch R, Martinez-Gonzalez MA, Corella D, Salas-Salvado J, Ruiz-Gutierrez

11 V, Covas MI, et al. Effects of a Mediterranean-style diet on cardiovascular risk factors: a 12 randomized trial. Ann Intern Med. 2006;145(1):1-11.

13 2. Sofi F, Abbate R, Gensini GF, Casini A. Accruing evidence on benefits of 14 adherence to the Mediterranean diet on health: an updated systematic review and meta15 analysis. Am J Clin Nutr. 2010;92(5):1189-96.

16 3. Martinez-Gonzalez MA, Garcia-Arellano A, Toledo E, Salas-Salvado J, Buil17 Cosiales P, Corella D, et al. A 14-item Mediterranean diet assessment tool and obesity 18 indexes among high-risk subjects: the PREDIMED trial. PLOS ONE. 2012;7(8):e43134.

19 4. Willett WC, Sacks F, Trichopoulou A, Drescher G, Ferro-Luzzi A, Helsing E, et 20 al. Mediterranean diet pyramid: a cultural model for healthy eating. Am J Clin Nutr. 21 1995;61(6 Suppl):1402s-6s.

22 5. George ES, Kucianski T, Mayr HL, Moschonis G, Tierney AC, Itsiopoulos C. A 23 Mediterranean diet model in australia: Strategies for translating the traditional 24 Mediterranean diet into a multicultural setting. Nutrients. 2018;10(4).

25 6. Ghanbari R, Anwar F, Alkharfy KM, Gilani AH, Saari N. Valuable nutrients and 26 functional bioactives in different parts of olive (Olea europaea L.)-a review. Int J Mol 27 Sci. 2012;13(3):3291-340.

28 7. International Olive Oil Council. Designations and definitions of olive oil 2018 29 [15/06/2018]. Available from: http://www.internationaloliveoil.org/estaticos/view/8330 designations-and-definitions-of-olive-oils.

31 8. Standards Australia. AS 5264-2011: Olive oils and olive-pomace oils. Standards 32 Australia Limited. 2011

33 9. George ES, Marshall S, Mayr HL, Trakman GL, Tatucu-Babet OA, Lassemillante 34 AM, et al. The effect of high-polyphenol extra virgin olive oil on cardiovascular risk 35 factors: a systematic review and meta-analysis. Crit Rev Food Sci Nutr. 2018:1-138.

36 10. Obied HK, Prenzler PD, Omar SH, Ismael R, Servili M, Esposto S, et al. Chapter 37 Six - Pharmacology of Olive Biophenols. In: Fishbein JC, editor. Advances in Molecular 38 Toxicology. 6: Elsevier; 2012. p. 195-242.

39 11. Santangelo C, Filesi C, Vari R, Scazzocchio B, Filardi T, Fogliano V, et al. 40 Consumption of extra-virgin olive oil rich in phenolic compounds improves metabolic 
1 control in patients with type 2 diabetes mellitus: a possible involvement of reduced levels

2 of circulating visfatin. J Endocrinol Invest. 2016;39(11):1295-301.

3 12. Ruano J, Lopez-Miranda J, Fuentes F, Moreno JA, Bellido C, Perez-Martinez P, 4 et al. Phenolic content of virgin olive oil improves ischemic reactive hyperemia in 5 hypercholesterolemic patients. J Am Coll Cardiol. 2005;46(10):1864-8.

6 13. Covas MI, Nyyssonen K, Poulsen HE, Kaikkonen J, Zunft HJ, Kiesewetter H, et 7 al. The effect of polyphenols in olive oil on heart disease risk factors: a randomized trial. 8 Ann Intern Med. 2006;145(5):333-41.

9 14. Farras M, Castaner O, Martin-Pelaez S, Hernaez A, Schroder H, Subirana I, et al. 10 Complementary phenol-enriched olive oil improves HDL characteristics in 11 hypercholesterolemic subjects. A randomized, double-blind, crossover, controlled trial. 12 The VOHF study. Mol Nutr Food Res. 2015;59(9):1758-70.

13 15. Lewington S, Whitlock G, Clarke R, Sherliker P, Emberson J, Halsey J, et al. 14 Blood cholesterol and vascular mortality by age, sex, and blood pressure: a meta-analysis 15 of individual data from 61 prospective studies with 55,000 vascular deaths. Lancet. 16 2007;370(9602):1829-39.

17 16. Triolo M, Annema W, Dullaart RP, Tietge UJ. Assessing the functional properties 18 of high-density lipoproteins: an emerging concept in cardiovascular research. Biomark 19 Med. 2013;7(3):457-72.

20 17. Khera AV, Cuchel M, de la Llera-Moya M, Rodrigues A, Burke MF, Jafri K, et 21 al. Cholesterol efflux capacity, high-density lipoprotein function, and atherosclerosis. N 22 Engl J Med. 2011;364(2):127-35.

23 18. Martinez-Lapiscina EH, Clavero P, Toledo E, San Julian B, Sanchez-Tainta A, 24 Corella D, et al. Virgin olive oil supplementation and long-term cognition: the 25 PREDIMED-NAVARRA randomized, trial. J Nutr Health Aging. 2013;17(6):544-52. 19. Marx W, Kelly JT, Marshall S, Cutajar J, Annois B, Pipingas A, et al. Effect of resveratrol supplementation on cognitive performance and mood in adults: a systematic literature review and meta-analysis of randomized controlled trials. Nutr Rev. 2018:nuy010-nuy.

20. Chan A-W, Tetzlaff JM, Altman DG, Laupacis A, Gøtzsche PC, Krleža-Jerić K, et al. SPIRIT 2013 statement: defining standard protocol items for clinical trials. Ann Intern Med. 2013;158(3):200-7.

21. Pipingas A, Harris E, Tournier E, King R, Kras M, Stough C. Assessing the Efficacy of Nutraceutical Interventions on Cognitive Functioning in the Elderly Curr Top Nutraceutical Res. 2010;8(2/3):79-87.

22. Massee LA, Ried K, Pase M, Travica N, Yoganathan J, Scholey A, et al. The acute and sub-chronic effects of cocoa flavanols on mood, cognitive and cardiovascular health in young healthy adults: a randomized, controlled trial. Front Pharmacol. 2015;6:93.

23. Pase MP, Grima N, Cockerell R, Stough C, Scholey A, Sali A, et al. The effects of long-chain omega-3 fish oils and multivitamins on cognitive and cardiovascular function: a randomized, controlled clinical trial. J Am Coll Nutr. 2015;34(1):21-31.

24. Kennedy G, Meyer D, Hardman RJ, Macpherson H, Scholey AB, Pipingas A. Physical Fitness and Aortic Stiffness Explain the Reduced Cognitive Performance Associated with Increasing Age in Older People. J Alzheimers Dis. 2018;63(4):1307-16. 25. Australian Institute of Health Welfare. The Active Australia Survey: A Guide and Manual for Implementation, Analysis and Reporting: Australian Institute of Health and Welfare; 2003. 
1 26. Hernaez A, Fernandez-Castillejo S, Farras M, Catalan U, Subirana I, Montes R, 2 et al. Olive oil polyphenols enhance high-density lipoprotein function in humans: a 3 randomized controlled trial. Arterioscler Thromb Vasc Biol. 2014;34(9):2115-9.

4 27. Sofi F, Casini A. Mediterranean diet and non-alcoholic fatty liver disease: new 5 therapeutic option around the corner? World J Gastroenterol. 2014;20(23):7339-46.

6

7

8

9

10

11

12 
Figure 1. Study flow

This is a post-peer-review, pre-copyedit version of an article published in Nutrition and Dietetics.

The final authenticated version is available online at: https://doi.org/10.1111/1747-0080.12531. 
Table 1. Study eligibility criteria

Inclusion criteria

- Aged 18-75 years

- $\quad$ BMI between $18.5-40 \mathrm{~kg} / \mathrm{m}^{2}$

Exclusion criteria

- Non-English-speaking persons

- Pregnant or lactating women

- History of adverse reactions to olive oil

- A habitual diet with $\geq 1$ tablespoons of olive oil per day

- Individuals following special diets vegetarian, coeliac, weight loss programs etc.

- Diagnosed with any of the following conditions: hyperlipidaemia; diabetes mellitus; hypertension; inflammatory conditions (e.g. rheumatoid arthritis), intestinal disease (e.g. inflammatory bowel disease; irritable bowel syndrome), food intolerances, blood coagulation disorders, any cognitive or mood disorder, any other physiological condition or disease that could impair adherence.

- Currently prescribed warfarin, anti-coagulant therapy, statin medications, all oral hypoglycaemic agents, insulin, immunosuppressant agents, antihypertensive agents, and nonsteroidal anti-inflammatory drugs, hormone replacement therapy, antibiotics

- Inability to cease nutrient supplement consumption, with the exception of iron, calcium and vitamin $\mathrm{D}$, for the trial duration

- Unstable body weight with $\geq 5 \mathrm{~kg}$ weight fluctuations in the prior 3 months.

- Special diet for medical reasons (e.g. gluten free for coeliac disease)

- Current smoker 
Exclusion criteria for the cognition testing component only

- Currently prescribed anti-depressant medication. 
Table 2: Study procedure and time points in the OLIVAUS trial

\begin{tabular}{|c|c|c|c|c|c|c|c|}
\hline Study procedure & T0 & T1 & $\mathrm{T} 2$ & T3 & $\mathrm{T} 4$ & T5 & T6 \\
\hline $\begin{array}{l}\text { Timepoint } \\
\text { description }\end{array}$ & $\begin{array}{l}\text { 2-weeks pre- } \\
\text { baseline }\end{array}$ & $\begin{array}{l}\text { Baseline, } \\
\text { commencement of } \\
\text { first intervention }\end{array}$ & $\begin{array}{l}\text { Mid- } \\
\text { intervention } \\
\text { contact }\end{array}$ & $\begin{array}{l}\text { End of first } \\
\text { intervention } \\
\text { (3-weeks post } \\
\text { T1 and start of } \\
\text { washout } \\
\text { period) }\end{array}$ & $\begin{array}{l}\text { Commencement of } \\
\text { second intervention } \\
\text { and end of washout } \\
\text { period }\end{array}$ & $\begin{array}{l}\text { Mid- } \\
\text { intervention } \\
\text { contact }\end{array}$ & $\begin{array}{l}\text { End of first } \\
\text { intervention } \\
\text { (3-weeks post } \\
\text { T3) }\end{array}$ \\
\hline $\begin{array}{l}\text { Total duration of } \\
\text { study procedures }\end{array}$ & 30 minutes & 1 hour & 10 minutes & 1 hour & 1 hour & 10 minutes & 30 minutes \\
\hline $\begin{array}{l}\text { Method of data } \\
\text { collection }\end{array}$ & $\begin{array}{l}\text { Telephone } \\
\text { OR Patient } \\
\text { interview }\end{array}$ & Patient interview & $\begin{array}{l}\text { Telephone } \\
\text { interview }\end{array}$ & $\begin{array}{l}\text { Patient } \\
\text { interview }\end{array}$ & Patient interview & $\begin{array}{l}\text { Telephone } \\
\text { interview }\end{array}$ & $\begin{array}{l}\text { Patient } \\
\text { interview }\end{array}$ \\
\hline $\begin{array}{l}\text { Screened \& } \\
\text { consented }\end{array}$ & $\checkmark *$ & & & & & & \\
\hline
\end{tabular}




\begin{tabular}{|c|c|c|c|c|c|c|}
\hline $\begin{array}{l}\text { Participant } \\
\text { characteristics }\end{array}$ & $\checkmark$ & & & & & \\
\hline $\begin{array}{l}\text { Delivery of 3-week } \\
\text { olive oil }\end{array}$ & $\checkmark$ & & & $\checkmark$ & & \\
\hline $\begin{array}{l}\text { Delivery of } \\
\text { Participant } \\
\text { Booklet }\end{array}$ & $\checkmark$ & & & $\checkmark$ & & \\
\hline $\begin{array}{l}\text { Intervention } \\
\text { consumed }\end{array}$ & $\checkmark$ & $\checkmark$ & $\checkmark$ & $\checkmark$ & $\checkmark$ & $\checkmark$ \\
\hline HDL-efflux & $\checkmark$ & & $\checkmark$ & $\checkmark$ & & $\checkmark$ \\
\hline $\begin{array}{l}\text { Total, HDL, LDL, } \\
\text { triglyceride levels }\end{array}$ & $\checkmark$ & & $\checkmark$ & $\checkmark$ & & $\checkmark$ \\
\hline Oxidized LDL & $\checkmark$ & & $\checkmark$ & $\checkmark$ & & $\checkmark$ \\
\hline hsCRP & $\checkmark$ & & $\checkmark$ & $\checkmark$ & & $\checkmark$ \\
\hline Blood pressure & $\checkmark$ & & $\checkmark$ & $\checkmark$ & & $\checkmark$ \\
\hline
\end{tabular}




\begin{tabular}{|l|c|c|c|c|c|c|}
\hline Arterial stiffness & $\checkmark$ & & $\checkmark$ & $\checkmark$ & $\checkmark$ \\
\hline Dietary intake & & $\checkmark$ & & $\checkmark$ & $\checkmark$ & $\checkmark$ \\
\hline Cognitive & $\checkmark$ & & $\checkmark$ & $\checkmark$ & $\checkmark$ \\
\hline Performance & & & & & $\checkmark$ \\
\hline Anthropometry & $\checkmark$ & & $\checkmark$ & $\checkmark$ & $\checkmark$ \\
\hline Adverse events & & $\checkmark$ & $\checkmark$ & $\checkmark$ & $\checkmark$ & $\checkmark$ \\
\hline Blinding & $\checkmark$ & & & & $\checkmark$ \\
\hline assessment & & & & & & $\checkmark$ \\
\hline
\end{tabular}

HDL, High Density Lipoprotein; hsCRP, high sensitivity C-Reactive Protein; LDL, Low Density Lipoprotein; 
Table 3. Polyphenol composition of the HPOO and LPOO provided in the OLIVAUS trial

\begin{tabular}{|c|c|c|c|}
\hline Biophenols & Units & HPOO & LPOO \\
\hline Hydroxytyrosol & $\mathrm{mg} / \mathrm{kg}$ & 3.3 & 5.3 \\
\hline Tyrosol & $\mathrm{mg} / \mathrm{kg}$ & 2.5 & 5.1 \\
\hline $\begin{array}{l}\text { Vanillic acid + } \\
\text { Caffeic acid }\end{array}$ & $\mathrm{mg} / \mathrm{kg}$ & 3.5 & 0.0 \\
\hline Vanillin & $\mathrm{mg} / \mathrm{kg}$ & 2.9 & 0.6 \\
\hline p-Coumaric Acid & $\mathrm{mg} / \mathrm{kg}$ & 13.0 & 1.0 \\
\hline $\begin{array}{l}\text { Hydroxytyrosol } \\
\text { Acetate }\end{array}$ & $\mathrm{mg} / \mathrm{kg}$ & 0.0 & 0.0 \\
\hline Ferulic acid & $\mathrm{mg} / \mathrm{kg}$ & 9.8 & 0.8 \\
\hline o-Coumaric Acid & $\mathrm{mg} / \mathrm{kg}$ & 0.0 & 0.0 \\
\hline $\begin{array}{l}\text { Decarb. } \\
\text { oleuroaglycone, Ox Al }\end{array}$ & $\mathrm{mg} / \mathrm{kg}$ & 6.5 & 2.7 \\
\hline Oleacein & $\mathrm{mg} / \mathrm{kg}$ & 71.7 & 6.3 \\
\hline Oleuropein & $\mathrm{mg} / \mathrm{kg}$ & 17.2 & 1.0 \\
\hline Oleuro aglycone, $\mathrm{Al}$ & $\mathrm{mg} / \mathrm{kg}$ & 11.0 & 0.8 \\
\hline Tyrosol Acetate & $\mathrm{mg} / \mathrm{kg}$ & 2.9 & 0.1 \\
\hline $\begin{array}{l}\text { Decarb. } \\
\text { Ligstraglycone, Ox Al }\end{array}$ & $\mathrm{mg} / \mathrm{kg}$ & 11.6 & 3.8 \\
\hline Oleocanthal & $\mathrm{mg} / \mathrm{kg}$ & 29.5 & 11.2 \\
\hline Pinoresinol + 1 & $\mathrm{mg} / \mathrm{kg}$ & 26.0 & 5.5 \\
\hline
\end{tabular}




\begin{tabular}{|c|c|c|c|}
\hline Acetoxy pinore & & & \\
\hline Cinnamic Acid & $\mathrm{mg} / \mathrm{kg}$ & 3.7 & 2.0 \\
\hline $\begin{array}{l}\text { Ligstroside } \\
\text { aglycone, Al }\end{array}$ & $\mathrm{mg} / \mathrm{kg}$ & 3.2 & 0.4 \\
\hline $\begin{array}{l}\text { Oleuro aglycone, } \\
\text { Ox Al Hy }\end{array}$ & $\mathrm{mg} / \mathrm{kg}$ & 13.8 & 3.0 \\
\hline Luteolin & $\mathrm{mg} / \mathrm{kg}$ & 13.9 & 1.8 \\
\hline $\begin{array}{l}\text { Oleuro aglycone, } \mathrm{Al} \\
\text { Нy }\end{array}$ & $\mathrm{mg} / \mathrm{kg}$ & 44.6 & 3.0 \\
\hline $\begin{array}{l}\text { Ligstro aglycone, } \\
\text { Ox Al Hy }\end{array}$ & $\mathrm{mg} / \mathrm{kg}$ & 6.4 & 1.4 \\
\hline Apigenin & $\mathrm{mg} / \mathrm{kg}$ & 9.3 & 1.0 \\
\hline Methyl-Luteolin & $\mathrm{mg} / \mathrm{kg}$ & 5.0 & 3.0 \\
\hline $\begin{array}{l}\text { Ligstroside } \\
\text { aglycone, Al Hy }\end{array}$ & $\mathrm{mg} / \mathrm{kg}$ & 9.0 & 7.9 \\
\hline $\begin{array}{l}\text { Total Biophenols - } \\
\text { HPLC }\end{array}$ & $\mathrm{mg} / \mathrm{kg}$ & 320.3 & 86.4 \\
\hline
\end{tabular}

HPOO, High polyphenol olive oil; HPLC, High performance liquid chromatography; LPOO, low polyphenol olive oil 